\title{
FAMILIA Y SOCIALIZACIÓN POLÍTICA. LA TRANSMISIÓN DE ORIENTACIONES IDEOLÓGICAS EN EL SENO DE LA FAMILIA ESPAÑOLA $^{1}$
}

\author{
Antonio M. Jaime Castillo \\ Universidad de Granada \\ E-mail: amjaime@ugr.es
}

\begin{abstract}
RESUMEN
La socialización política básica tiene lugar en el seno de la familia. Los estudios clásicos de socialización política han mostrado que los hijos tienden a reproducir las orientaciones ideológicas de sus padres. Los datos para el caso español muestran una gran similitud entre la posición política de la generación actual y la anterior. A este respecto, cabe señalar que, a pesar de las transformaciones experimentadas por la familia española en las últimas décadas, ésta sigue siendo una instancia eficaz en el proceso de socialización política. Finalmente se analiza la influencia de algunas variables sobre la distancia ideológica entre padres e hijos.
\end{abstract}

\section{INTRODUCCIÓN}

Pocas veces al analizar el comportamiento electoral y otros fenómenos políticos se le presta atención a la familia. No ocurría así, no obstante, en la teoría política clásica, en cuya tradición ha habido tratadistas que han reservado un lugar preferente para el estudio de la familia. Así, por ejemplo, Aristóteles titulaba el Primer Libro de la Política "Comunidad Política y Comunidad Familiar». Esto tenía todo su sentido en la polis griega, en cuya praxis política no

1 El autor agradece los valiosos comentarios y sugerencias de los Profesores Julio Iglesias de Ussel y Diego Ruiz Becerril a la versión preliminar de este trabajo. No obstante, asume toda la responsabilidad por los errores y omisiones contenidos en esta versión definitiva. 
existía una división clara entre el mundo de lo público y lo privado, sino que la política estaba orientada a la realización de un determinado concepto de «vida buena», cuyo contenido ético holístico imbricaba a las esferas pública y privada de la vida del ciudadano.

Sin embargo, el liberalismo decimonónico, no tanto en la versión kantiana como en la versión utilitarista de los Bentham, Mills y otros, va a establecer la separación tajante entre las esferas de la vida pública y la vida privada. Del mismo modo que la revolución industrial habría de suponer la disociación entre la producción y el núcleo familiar, la revolución liberal apartó la política de la vida familiar. Daniel Bell (1994) ha sabido como nadie ilustrar las contradicciones que emergen entre los distintos subsistemas sociales de la civilización capitalista (economía, política y cultura), puesto que cada uno de éstos se rige por un principio axiológico propio y que entra en contradicción con los demás, abocando a la sociedad capitalista a la crisis.

Gran parte de la moderna investigación política empírica ha estado marcada por ese sesgo de considerar a la política como una actividad pública, desconectada de la vida privada. El comportamiento político, no obstante, está marcado por determinantes de índole privada. Se halla condicionado por las experiencias aprendidas y transmitidas en el seno de la familia. Las actitudes políticas básicas se forman en los procesos de socialización que tienen lugar en la infancia temprana, en contacto con la familia (Abramson, 1983; Dowse y Hughes, 1999; Easton y Dennis, 1967, 1969; Greenstein, 1965; Greenstein y Tarrow, 1970; Hess y Torney, 1965; Hyman, 1959; Jennings y Niemi, 1968, 1974, 1981). En el caso concreto del comportamiento electoral, la familia juega un papel fundamental en la transmisión de las lealtades partidistas, las cuales se encuentran en la base de la formación de las decisiones electorales de los individuos adultos, según argumenta buena parte de la teoría contemporánea sobre el comportamiento electoral. Esta continuidad ideológica entre padres e hijos fue formulada por Lane (1959) como la «ley de Mendel de la política».

En sentido general, puede decirse que la familia es el grupo básico de la sociedad. En su seno se produce el nacimiento e inserción del individuo en la vida social, así como la transmisión de las normas y valores fundamentales para la comunidad. Es «el primer núcleo de transmisión de valores sociales» (Iglesias de Ussel, 1990: 236). A pesar de que en la actualidad la familia haya cedido parte de sus funciones como agente socializador, en favor de instituciones especializadas, como la escuela o los medios de comunicación de masas, en modo alguno puede minusvalorarse su importancia.

$\mathrm{Y}$ ello, por dos razones fundamentales. De una parte, porque el primer grupo de pertenencia del recién nacido es la familia. Esta circunstancia introduce un importante condicionante sobre la personalidad individual, que vendrá marcada de forma decisiva por las vivencias en los primeros estadios vitales. Por otro lado, la familia conserva hoy día un alto grado de aceptación social, como muestran los indicadores sobre valoración de esta institución y 
por la prolongada permanencia de la convivencia entre padres e hijos, incluso cuando éstos se hacen adultos.

La influencia de la familia no pasó por alto para una importante corriente de investigación del comportamiento político, cuyo enfoque suele ser conocido como "modelo de identificación partidaria» y que se desarrolló en los años cincuenta en Estados Unidos, en el Center for Political Estudies de la Universidad de Michigan. En esencia, viene a proponer que la decisión de voto de un elector concreto viene determinada por las afinidades de este elector con respecto a los distintos partidos en liza en la elección ${ }^{2}$. Esas afinidades ideológicas se forman durante el proceso de socialización, en el cual el papel central corresponde a la familia. Cabe esperar, por tanto, que las afinidades ideológicas se transmitan de padres a hijos, de forma que los individuos que nacen en el seno de una familia con una determinada orientación política reproduzcan en su época adulta esa misma inclinación política.

Este es el marco en el que se inscribe este trabajo, cuyo objeto es conocer el grado en que las preferencias ideológicas se transmiten en España en el seno de la familia, o hasta qué punto, la familia española cumple un papel eficiente como instancia de socialización política. La hipótesis de partida será que las preferencias ideológicas se transmiten, fundamentalmente, en el seno de la familia.

A continuación, en el próximo epígrafe, se tratará brevemente de la intervención de la familia en el proceso de socialización política. Después se expondrán sucintamente las implicaciones del enfoque de identificación partidaria, así como los estudios clásicos en el campo de la transmisión de las identidades ideológicas de padres a hijos. Y, por último, se analizarán los datos de transmisión de identidades ideológicas entre padres e hijos en la sociedad española, con el objetivo de comprobar hasta qué punto se cumplen las hipótesis desarrolladas por los estudios de socialización política.

\section{LA FAMILIA Y EL PROCESO DE SOCIALIZACIÓN POLÍTICA}

La unidad familiar es el primer habitáculo de socialización del individuo. Como señala Félix Ortega, los padres son un influjo permanente y un modelo a imitar para los hijos. Así es como se proyectan sobre los miembros más jóvenes de la comunidad una serie de «cualidades, cuadros valorativos y orientaciones vitales» (Ortega, 1975: 175). Desde este punto de vista, el estudio de la familia adquiere una enorme relevancia para la comprensión de la dimensión ideológica y política en una determinada sociedad, al tiempo que es una insti-

${ }^{2}$ Las bases teóricas y metodológicas de este enfoque pueden encontrarse en dos obras centrales de la Sociología y la Ciencia Política de la segunda mitad del siglo XX: A. CAmpbell, P. E. Converse, W. E. Miller y D. E. Stokes (1960): The American voter, Wiley, New York; N. H. Nie, S. Verba y J. R. PetrociK (1976): The changing American voter, Harvard University Press, Cambridge. 
tución política de enorme importancia (Iglesias de Ussel, 1990). Tanto más cierto en un país como España, en donde, según señalaba el Profesor Murillo Ferrol, «escasean las instancias de socialización de auténtico nivel comunitario, por encima de la familia» (Murillo Ferrol, 1987: 298).

Desde siempre, los pensadores sociales vieron en la familia el garante de la moral pública y privada. Entre los clásicos de la teoría sociológica contemporánea, Talcott Parsons, al analizar el sistema social desde su esquema A.G.I.L. otorgó la función sistémica de latencia a la familia ${ }^{3}$. Parsons distinguía entre la familia de orientación y la familia de procreación. En la primera es donde el individuo adquiere las normas y valores del sistema social (Parsons, 1986; Parsons y Bales, 1955). No cabe exagerar la importancia que Parsons atribuía a la familia, debido, en parte, al conocido énfasis de las teorías funcionalistas en la prioridad de los valores como fuente del consenso normativo, base de todo sistema social.

La investigación específica en el campo de la socialización política también ha subrayado la enorme importancia de la familia en el proceso de adquisición de normas políticas. A pesar de que el tema de la transmisión de actitudes políticas en el seno de la familia ha sido objeto de cierta controversia entre los especialistas, existe un consenso generalizado en que la familia de origen moldea las orientaciones ideológicas básicas de los individuos (Percheron y Jennings, 1981). Tanto los modelos de orientación psicoanalítica, como los de orientación cognoscitiva ${ }^{4}$, implican una presencia activa de la familia en el proceso de socialización política, puesto que, como señalan Dowse y Hughes, «la primera etapa de la socialización en todas las sociedades suele tener lugar dentro de la familia o en el marco del grupo de parentesco o iguales» (Dowse y Hughes, 1990: 231).

Las grandes transformaciones que ha experimentado la familia y las prácticas de crianza en las últimas décadas en las sociedades occidentales, como las que se derivan de la incorporación de la mujer al mercado laboral, no han debilitado la capacidad de influencia de la familia sobre el proceso de socialización política. Annick Percheron (1990) apunta que el nivel de comunicación dentro de la familia ha permanecido estable. No obstante, según los datos que maneja el propio autor, la confianza entre padres e hijos ha tendido a aumentar, así como los temas, antes espinosos, en los que la comunicación entre padres e hijos es ahora más frecuente y menos conflictiva. La combinación de

${ }^{3}$ Esta función se refiere al mantenimiento y renovación de los patrones culturales en el seno de la sociedad, los cuales proporcionan las motivaciones privadas a los individuos.

${ }^{4}$ Por modelos de orientación psicoanalítica se entienden aquellos en los que la socialización política es vista como el proceso por el que los individuos aprenden a controlar sus impulsos antisociales. Por modelos de orientación cognoscitiva se hace referencia a aquellos que hacen hincapié en el proceso de aprendizaje de roles. Esta clasificación coincidiría, a grandes rasgos, con la que realiza Ángel Rodríguez, que divide los modelos en dos grupos, de conformidadlegitimación y de diferenciación-innovación. Vid. en A. RodRíGUEZ (1988): «Socialización política», en J. Seoane y A. Rodríguez (eds.), Psicología política, Pirámide, Madrid. 
varios factores y procesos de cambio en el seno de la familia hacen que las relaciones entre padres e hijos se funden sobre bases distintas a las de antaño. En las últimas décadas se ha producido un decaimiento pronunciado de la autoridad paterna dentro de las estructuras familiares (Caplow, 1995; Halman y Moor, 1994), que ha facilitado la mejoría de las relaciones intergeneracionales.

Ya no se espera de los hijos un apego incondicional a las ideas de sus padres, sino que se les forma para que desarrollen su propia identidad en el marco de una socialización mucho menos autoritaria. No obstante, ello no ha supuesto un distanciamiento de los hijos con respecto de los padres. Las transformaciones que ha experimentado la familia en las últimas décadas apuntan hacia una transmisión de valores más democrática en el seno de la familia, con más participación de los hijos. Pero ello no implica que la transmisión de normas y orientaciones de valor de padres a hijos sea menos efectiva.

La familia no ha permanecido aislada de la tendencia hacia la reducción en la importancia de la autoridad personal en las relaciones sociales, según pone de manifiesto Caplow (1995). En su interior también se ha incrementado la libertad personal, la igualación creciente en los roles sexuales, etc. La institución familiar, como puede verse, se va adaptando al nuevo marco social en el que se inserta. Pero no por ello ha dejado de cumplir sus funciones, sino que ahora las cumple de forma distinta. A su vez, la sociedad en su conjunto se ve afectada por estos cambios que tienen lugar en el nivel microsociológico.

En definitiva, la educación, los mecanismos y los procesos no han cambiado mucho en el fondo, sino que han cambiado en la forma. Hay un mayor reconocimiento de la autonomía de los hijos, que tienen el derecho a ser, pensar diferente, a pensar como ellos quieran. La tolerancia de los padres hacia ellos es muy grande en la actualidad y comparativamente mucho mayor que antes. Se trata de una socialización que es sin duda menos autoritaria, menos directiva y, por tanto, la transmisión de valores se hace mejor (Percheron, 1990).

\section{EL MODELO DE IDENTIFICACIÓN PARTIDARIA. UN MARCO TEÓRICO PARA LA TRANSMISIÓN DE AFINIDADES IDEOLÓGICAS}

Harrop y Miller (1990) señalan como primer presupuesto del modelo de identificación partidaria que la mayoría de los electores sienten una afinidad hacia un partido, la cual es heredada en el seno de la familia. Este enfoque explica el comportamiento de los votantes sobre la base de su identificación partidista, definida por las actitudes favorables o desfavorables hacia los diferentes partidos políticos. El origen último de la identificación partidista de los sujetos se halla en los procesos de socialización política, a través de los cuales se desarrolla un complejo sistema actitudinal de identificación o aversión hacia los distintos elementos del sistema político (Jaime Castillo, 1998). 
La importancia atribuida al concepto de afinidad ideológica en el marco de esta teoría llevó pronto a los investigadores a preguntarse acerca del proceso de formación de la misma. Como señala Abramson, «uno de los primeros hallazgos de la investigación sobre socialización política es que la mayoría de los americanos aprenden las lealtades partidistas de sus padres y que estas lealtades son aprendidas a una edad muy temprana» (Abramson, 1983: 86). Según sostenía Hyman (1959), la mayoría de la gente adquiere una afinidad ideológica aun antes de tener un conocimiento razonable sobre la política. De hecho, las afinidades ideológicas son un esquema de interpretación de la realidad política que ayuda a interpretar la información política nueva que se va recibiendo.

Este modelo tiene unas importantes implicaciones para el estudio y comprensión de los procesos políticos, puesto que ofrece una explicación acerca de la estabilidad de los sistemas políticos a lo largo del tiempo. Según el modelo desarrollado por Percheron y Jennings (1981), el esquema causal sería el siguiente: la transmisión paterna de las afinidades partidistas tiene como efecto la continuidad de las preferencias de una generación a la siguiente, lo cual se refleja en la estabilidad de las mismas. $Y$ esa estabilidad de las preferencias partidistas, a su vez, es la causa directa de la estabilidad del sistema de partidos.

Los estudios pioneros de Greenstein (1965) y de Easton y Hess (Hess y Torney, 1965), aportaron las primeras fuentes de evidencia empírica para sostener la hipótesis de la transmisión familiar de afinidades ideológicas. Según comprobaron estos autores, los niños norteamericanos tenían la capacidad de definirse como «demócratas» o «republicanos» a edades muy tempranas, incluso aunque su nivel de información sobre política fuera muy reducido. El estudio de Greenstein encontró, además, que apenas un número bastante bajo de niños se identificaban con un partido distinto a aquel con el que se identificaban sus padres.

El estudio empírico de mayor relevancia sobre socialización política en la década de los sesenta es el que realizó el Survey Research Center de la Universidad de Michigan en 1965, sobre una muestra nacional de 1.500 alumnos de Escuelas Superiores. A pesar de que los resultados del estudio deben ser analizados con cautela, debido a que no se utilizaron datos longitudinales ${ }^{5}$, la conclusión más evidente que se desprende del mismo es que existía una clara correspondencia entre las afinidades ideológicas de los estudiantes y las de sus padres. Por otro lado, el estudio también reveló que los estudiantes tenían un grado de identificación ideológica más débil que sus padres (Jennings y Niemi, 1968).

Aunque M. Kent Jennings y Richard G. Niemi no llegaron a explicar de forma convincente el porqué de esa debilidad de la identificación partidaria de los alumnos sí que proveen algunas conclusiones interesantes sobre la forma en

${ }^{5}$ Una discusión detallada de los principales problemas metodológicos de este estudio puede verse en P. R. Abramson (1983): Political attitudes in America. Formation and change, W. H. Freeman and Co., San Francisco. 
la que los hijos adquieren afinidades ideológicas de sus padres. La primera conclusión es que el éxito en la transmisión de las lealtades partidistas está en función del grado de politización del hogar. En aquellos hogares donde se discutía frecuentemente de política era más probable que los hijos tuvieran las mismas actitudes que los padres. Otro dato importante es que la transmisión era más difícil cuando uno de los padres no tenía adscripción partidaria y aún más difícil si cada uno de los padres era afín a un partido distinto.

En 1972, Kent L. Tedin (1974) condujo otro estudio de dimensiones más reducidas, cuyo objetivo era tratar de determinar la correlación entre las actitudes de los padres ante distintas cuestiones políticas de actualidad y las de los hijos. El hallazgo fundamental de Tedin fue que existía un alto grado de correspondencia entre las lealtades partidistas de los padres y los hijos, pero que la relación entre las preferencias por distintos tipos de políticas públicas entre padres e hijos era bastante menor.

Un estudio internacional llevado a cabo en Estados Unidos, Holanda, Gran Bretaña, Alemania Occidental y Austria en 1974 reveló igualmente que los hijos tendían a tener un grado de identificación partidista más débil que los padres (Barnes y Kaase, 1979). La dimensión comparativa del estudio permitió comprobar algunas diferencias transnacionales. Las correlaciones más altas entre las afinidades ideológicas de padres e hijos fueron halladas en Austria, con un sistema bipartidista bastante polarizado, al tiempo que las más bajas se daban en Holanda, un país con un sistema multipartidista bastante complejo.

En 1975, Annick Percheron dirigió un estudio similar en Francia. Sobre la base de los resultados de este estudio, Percheron y Jennings (1981) trataban de refutar la tesis de Converse y Dupeux (1962) de que el sistema de partidos francés era inestable debido a la ausencia de transmisión intergeneracional de afinidades ideológicas. Sus datos mostraron que las continuidades ideológicas predominaban en Francia, ya que la correlación entre la posición ideológica de padres e hijos era similar al de otros países como Italia u Holanda. Y esas continuidades estaban enraizadas, en gran medida, «en la perpetuación de las tradiciones familiares» (Percheron y Jennings, 1981: 423).

A pesar de la evidencia empírica acumulada, el conocimiento sobre la forma en la que las normas políticas, en general, son transmitidas en el seno de la familia es bastante limitado. Existen bastantes indicios de que el proceso de aprendizaje de las afinidades ideológicas no se basa en un proceso de adoctrinamiento, sino que depende más bien de los comportamientos observados por el niño durante su etapa de socialización. Como señala Abramson (1983), la exitosa transmisión de identificaciones partidistas a través de generaciones haría depender los cambios de gobiernos de la tasa de natalidad entre los partidarios de uno u otro partido. En cualquier caso, la transmisión de afinidades ideológicas debe estar influida por fuerzas políticas que operan en el corto plazo, lo cual viene a mostrar que el proceso de transmisión intergeneracional permite tanto el cambio como la continuidad de las lealtades partidistas. 


\section{LAS CONTINUIDADES IDEOLÓGICAS ENTRE LAS GENERACIONES EN ESPAÑA}

Si bien no disponemos de estudios empíricos a través de los que se puedan analizar las pautas de continuidad en el comportamiento electoral entre unas generaciones y otras en España, numerosos estudios sobre la transición política revelan bien a las claras que la familia ha contribuido, de forma decisiva, a la transmisión de las adscripciones partidistas a lo largo del prolongado periodo de la dictadura franquista. Así, por ejemplo, se desprende del hecho de que los resultados de las primeras elecciones de la transición reprodujeran, de forma más o menos aproximada, la composición de fuerzas políticas que surgió en las últimas elecciones que tuvieron lugar durante la II República (Iglesias de Ussel, 1998; Maravall, 1985; Tezanos, 1982). Tal como ha señalado Maravall, los datos sobre la continuidad intergeneracional del electorado parecen indicar «una transmisión de ideas entre padres e hijos» (Maravall, 1985: 190).

Estas continuidades sólo fueron posibles porque las lealtades partidistas se transmitieron de padres a hijos en el seno de la familia durante un largo periodo de cuarenta años. De hecho cabe pensar que la propia naturaleza del régimen franquista contribuyó a que esto fuese así. Al quedar la política desterrada del debate público parece más probable que la discusión sobre cuestiones políticas fuera desplazada hacia un locus privado como la familia, en el que no existía posibilidad de censura. Todos los indicios llevan a la conclusión de que la familia jugó un papel muy importante en la transmisión de las lealtades partidistas durante el franquismo, y, como consecuencia, en la continuidad de las pautas de comportamiento electoral de una generación a otra.

Una de las cuestiones relacionadas con la transmisión de normas políticas en el seno de la familia, es el grado de comunicación que se produce en su interior. De acuerdo con la tesis de Jennings y Niemi, debemos esperar que la transmisión sea más efectiva cuando el grado de discusión política sea mayor. Numerosos estudios sobre la juventud española se han ocupado del tema tanto desde la perspectiva del nivel de comunicación como desde la perspectiva del consenso normativo.

Los resultados de diversos estudios muestran, en primer lugar, que el grado de discusión política en el seno de la familia española es relativamente bajo. Según datos del CIS de 1999, sólo el 26 por 100 de los jóvenes hablan de política con sus padres con mucha o bastante frecuencia (CIS, 1999). No obstante, este dato debe interpretarse dentro de un contexto específico. Por una parte, no es extraño desde el punto de vista comparado. En la mayoría de países de nuestro entorno se dan porcentajes similares. Por otro lado, este dato no refleja principalmente una falta de comunicación política en el seno de la familia, sino un básico y persistente desinterés por la política.

En 1991, en un estudio específico del Instituto de la Juventud (1991) sobre las actitudes políticas de los jóvenes españoles, se preguntaba a éstos a qué personas e instituciones solían acudir a comentar acontecimientos políti- 
cos. Entre las instituciones, un 20 por 100 señaló a la familia, frente a otras instituciones con más dimensión política (partidos o sindicatos), a las que sólo acudían un 2 por 100 de los jóvenes. Y entre las personas, un 45 por 100 acudían a alguno de los dos progenitores, siendo las chicas las más inclinadas a debatir sobre temas políticos con la familia.

Por otra parte, el consenso normativo entre padres e hijos ha crecido sustancialmente desde la transición hasta hoy. En 1981 eran un 26 por 100 los jóvenes de dieciocho a veinticuatro años los que manifestaban compartir opiniones políticas con los padres, mientras que esta proporción era del 32 por 100 en 1994 (Andrés Orizo, 1996). En 1991, el reseñado estudio del Instituto de la Juventud (1991) señalaba que un 56 por 100 de los jóvenes estaban de acuerdo con el padre, algunas veces o casi siempre, en temas políticos, siendo un 55 por 100 los que declaraban lo mismo con respecto a la madre. Y en el mismo sentido, según los datos del CIRES, la suma de los que estaban más bien de acuerdo y totalmente de acuerdo en política con sus padres representaban el 51 por 100 en 1993 y el 50 por 100 en $1994^{6}$.

En una escala de discrepancia normativa sobre distintos temas, que fue aplicada en los estudios de juventud de 1994 y 1999, la política aparecía como uno de los temas en los que los jóvenes veían menos distinta su forma de pensar de la de sus padres, sólo por detrás de la familia y el trabajo. En ambos años coincidía el hecho de que eran las chicas las que menos discrepaban de sus padres, los de menos nivel educativo y los que declaraban una mayor práctica religiosa (Elzo, 1999). En el citado estudio del Instituto de la Juventud (1991), un 19 por 100 de los jóvenes manifestaba compartir las preferencias electorales con el padre y un 12 por 100 con la madre. En el mismo estudio se reflejaba también que un 54 por 100 decían discutir la decisión de votar antes de unas elecciones dentro de la familia.

Si bien todos estos datos dan idea de que la familia tiene una influencia estable sobre la orientación política de los jóvenes españoles, sería necesario plantearse si este consenso valorativo es el resultado de los procesos de socialización política que se dan en el seno de la familia o, por el contrario, está determinado por factores ambientales que influyen a la vez sobre padres e hijos. La investigación a este respecto es bastante escasa en España, e incluso a nivel comparado.

No obstante, en el estudio de juventud de 1991 se preguntaba a los jóvenes qué personas e instituciones les habían influido más en la formación de sus actitudes políticas. Un 38 por 100 de los encuestados señaló al padre, mientras que un 27 por 100 señalaba a la madre. Así pues, según perciben los propios jóvenes españoles, los progenitores ejercen una influencia considerable en las orientaciones políticas de las nuevas generaciones. Lo curioso del dato es la importante diferencia entre padres y madres, que no suele darse en otras dimensiones relacionadas.

${ }^{6}$ Encuestas sobre Juventud del Centro de Investigaciones de la Realidad Social para 1993 y 1994. 
Respecto al tema concreto de las orientaciones ideológicas, se observa, a nivel agregado, una continuada convergencia en las orientaciones ideológicas de padres e hijos desde principios de los años ochenta hasta nuestros días, siempre visto desde la perspectiva de los hijos. Si en 1984 los jóvenes de quince a veinticuatro años se situaban, de media, en el 4,24 de una escala ideológica de 1 a 10 y ubicaban a sus padres en el punto 5,62; en 1999 los jóvenes se situaban en el punto 4.56 y situaban a los padres en el $5^{7}$. Este proceso de convergencia es fruto de una doble tendencia. Por una parte, los jóvenes han evolucionado hacia el centro desde la izquierda, mientras que los padres van evolucionando hacia el centro desde la derecha. Pero ha sido mucho mayor el desplazamiento realizado por los padres.

\section{LA TRANSMISIÓN DE ORIENTACIONES IDEOLÓGICAS EN EL SENO DE LA FAMILIA ESPAÑOLA}

En primer lugar, convendrá definir claramente qué se entiende aquí por afinidades ideológicas. Mientras que en el contexto de la democracia norteamericana los estudios sobre identidades políticas han mostrado que los ciudadanos se identifican, principalmente, con uno de los grandes partidos, republicano o demócrata, existen diversas evidencias que ponen de manifiesto que en Europa las afinidades políticas van dirigidas hacia posiciones ideológicas genéricas, derecha o izquierda, antes que hacia un partido concreto (Harrop y Miller, 1990).

Este hecho fue puesto de manifiesto también por Percheron y Jennings (1981), quienes mostraron que, en sistemas multipartidistas como el francés, sometidos a la variabilidad de las siglas políticas, resultaba difícil que cristalicen afinidades hacia partidos concretos. En el imaginario colectivo del electorado existirían dos grandes espacios políticos, identificados como izquierda y derecha, hacia los que se dirigen sus actitudes positivas o negativas. El proceso de socialización política está marcado por este marco conceptual y los niños adquieren sus actitudes políticas en relación con las esferas de izquierda y derecha.

España, en concreto, señala Pilar del Castillo (1990), es uno de los países con los niveles de afinidad hacia los partidos políticos más bajos en perspectiva comparada. Sin embargo, esto no se ha traducido en altas dosis de volatilidad del comportamiento electoral inter-grupos, pese a que sí se han dado considerables procesos de volatilidad intra-grupos ${ }^{8}$. La explicación a este

La escala izquierda-derecha es una escala de diferencial semántico, utilizada habitualmente para medir la posición ideológica de un individuo. En una escala de 10 puntos el 1 representa a la extrema izquierda y el 10 a la extrema derecha.

${ }^{8}$ A nivel conceptual, se entiende por volatilidad inter-grupos el cambio de voto de un partido del bloque de la izquierda hacia uno del bloque de la derecha o viceversa. La volatilidad intragrupos denota la transferencia de votos entre partidos del mismo bloque ideológico. 
hecho reside en que las orientaciones políticas de los españoles se dirigen hacia grandes ejes de valor, como izquierda-derecha, antes que hacia partidos políticos concretos. Algunos autores han llegado a hablar, en este sentido, de un "cleavage» del electorado español en torno al eje izquierda-derecha (Montero, 1996).

Por este motivo, si bien en los estudios sobre transmisión de actitudes políticas en Estados Unidos se acostumbra a tomar como indicador la coincidencia entre padres e hijos en la identificación con uno de los partidos, en este trabajo se tomará como indicador la similitud entre la ubicación ideológica de padres e hijos sobre un continuum izquierda-derecha. Para este fin se utilizarán los datos de cinco encuestas sobre "Cultura Política y Económica» realizadas por el Centro de Investigaciones de la Realidad Social entre 1992 y 1996. En estos estudios se les pedía a una muestra representativa de españoles mayores de dieciocho años que se ubicaran en una escala de diferencial semántico izquierdaderecha a sí mismos y a sus padres?.

Es obvio el hecho de que los respondentes pueden sesgar, en alguna magnitud difícil de precisar a priori, la ubicación ideológica de sus padres, pues su percepción habría de ser siempre relativa. Tanto más en aquellas familias en las que los temas políticos fuesen poco tratados. Sin embargo, no existen en España datos sobre familias completas, similares a los datos de panel de Estados Unidos, que puedan utilizarse para estos fines. Y, aun siendo éste un indicador imperfecto, puede ayudar a entender el papel de la familia española en la transmisión de las lealtades ideológicas.

En otro orden de cosas, tampoco hemos de olvidar que el hecho de que padres e hijos tengan actitudes similares no explica el proceso por el que los niños aprenden las actitudes políticas. De hecho no existe una garantía de que esa similitud en las orientaciones ideológicas sea producto del aprendizaje dentro de la familia. Puesto que padres e hijos comparten el mismo ambiente social y cultural, siempre podríamos esperar alguna similitud ideológica, incluso aunque no hubiese comunicación en el seno de la familia (Jennings y Niemi, 1974).

A pesar de ser ésta una objeción bastante cualificada, la evidencia empírica acumulada sugiere que las orientaciones ideológicas anteceden a la formación de otras actitudes sobre el ambiente social en la infancia temprana. En efecto, los primeros estudios sobre el proceso de socialización política en Estados Unidos, mostraron que los niños adquieren sus afinidades ideológicas a edades verdaderamente tempranas (Davies, 1970). En el estudio de Greenstein, de 1958, seis de cada diez alumnos de escuelas primarias (de entre siete y catorce años) mostraban una preferencia claramente definida por algún partido (Greenstein, 1965). El estudio de Easton y Hess reveló que incluso entre los niños de segundo grado de primaria (de siete años) uno de cada tres tenía una preferen-

${ }^{9}$ Esta escala es de siete puntos, en donde 1 significa extrema izquierda y 7 significa extrema derecha. 
cia ideológica clara (Hess y Torney, 1965). Y Schwartz (1975) señala que las orientaciones ideológicas básicas se desarrollan entre los tres y los seis años, o incluso antes.

En perspectiva comparada, el caso español muestra niveles de asociación entre la posición ideológica de los padres y la de los hijos bastante similares a los de otros países como Italia o Francia ${ }^{10}$, aunque inferiores a los de Alemania o Inglaterra ${ }^{11}$. El motivo de estas diferencias transnacionales tiene más relación con los diferentes sistemas políticos y la cultura política general de estos países, antes que con la fortaleza de sus estructuras familiares. Porque de lo contrario nos veríamos obligados a concluir que las relaciones familiares son más débiles en los países mediterráneos, cosa que resulta contradictoria con la evidencia empírica.

En el caso español, en concreto, concurren ciertos elementos que ayudan a explicar esa menor intensidad de la asociación entre la ideología de las generaciones sucesivas. Por una parte, los bajos niveles de interés por la política típicos de nuestra cultura política deben incidir en el grado de comunicación sobre temas políticos en el seno de la familia, lo cual contribuye a una menor transmisión de orientaciones ideológicas. Otro elemento tiene su origen en las características del sistema de partidos español, que está atravesado por distintos subsistemas de partidos regionales. Estos partidos están caracterizados ideológicamente sobre el eje nacionalismo-centralismo antes que sobre el eje izquierda-derecha. Por este motivo, las pautas de socialización política están marcadas por distintas dimensiones ideológicas, que no pueden sintetizarse en la escala izquierda-derecha.

En la tabla 1 se pueden ver los coeficientes $t a u-b$, que miden la correlación entre la ideología del encuestado y la de ambos progenitores entre 1992 y $1996^{12}$. El valor de los coeficientes de correlación entre la ideología propia y la del padre oscilan entre 0,37 en 1995 y 0,49 en 1994. La correlación con la ideología de la madre oscila entre 0,39 en 1995 y 0,51 en 1993. La variación en los niveles de correlación no responde, sin embargo, a ningún patrón de cambio a lo largo del tiempo, en sentido ascendente o descendente. La estabilidad de los datos es consecuencia de que las pautas de socialización cambian a un ritmo lento en cada sociedad y esos cambios sólo pueden percibirse al comparar generaciones distintas.

En todos los años las correlaciones entre la posición ideológica propia y la de la madre son ligeramente superiores a las existentes con la del padre. Cabe suponer que, a pesar de los cambios en las pautas de crianza derivadas, fundamentalmente, de la progresiva incorporación de la mujer al mercado de traba-

${ }^{10}$ Según los datos presentados por A. Percheron y M. K. Jennings (1981): «Political continuities in French families», en Comparative Politics, núm. 13, pp. 421-436.

${ }^{11}$ Los datos para estos países pueden verse en S. H. BARnes, M. KAASE et al. (1979): Political action: Mass participation in five Western Democracies, Sage Publications, Beverly Hills.

${ }^{12}$ El coeficiente tau-b es una medida de asociación para variables ordinales, que se calcula a partir de los rangos de pares de casos en dos variables. 
TABLA 1

Correlaciones (tau-b) entre la posición ideológica propia y la de ambos progenitores*

\begin{tabular}{|c|c|c|c|c|c|}
\hline & 1992 & 1993 & 1994 & 1995 & 1996 \\
\hline 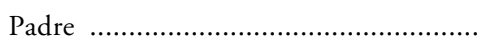 & 0,42 & 0,48 & 0,49 & 0,37 & 0,47 \\
\hline 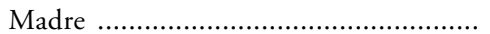 & 0,45 & 0,51 & 0,50 & 0,39 & 0,48 \\
\hline
\end{tabular}

* Todas las correlaciones son significativas para un nivel de confianza superior al 99 por 100.

Fuente: Elaboración propia, a partir de las Encuestas de Cultura Política y Económica del CIRES (1992-1996).

jo, la mujer sigue teniendo un papel más importante en el proceso de socialización. Durante los primeros estadios de la infancia, los niños pasan más tiempo en contacto con la madre. Y así, los niños adquieren más normas políticas de la madre. Más aún, esta mayor proximidad con la madre parece darse en todos los aspectos durante la etapa adulta de los individuos. Bahr, Déchaux y Stiehr (1995), analizando los vínculos entre padres e hijos adultos, subrayan que este hecho está relacionado con la erosión de la figura paterna y el debilitamiento de su concepto de autoridad tradicional.

Otra cuestión de interés es la dirección y evolución de la distancia ideológica entre padres e hijos, sobre el continuum izquierda-derecha. Este indicador se construye restando a la posición ideológica de cada entrevistado la de cada uno de sus progenitores. Los valores negativos, por tanto, indican que la nueva generación se sitúa más a la izquierda que la precedente, mientras que los valores positivos indican desplazamientos hacia la derecha.

En el gráfico 1 se representa la media de la distancia ideológica con el padre entre los años 1992 y 1996. A simple vista se observa que las distancias son bastante reducidas. El valor modal de la distancia toma el valor cero todos los años. Y la media no llega nunca a la unidad. Según se desprende de los datos, un porcentaje que ronda el 40 por 100 de los españoles comparten la misma posición ideológica que su padre ${ }^{13}$. En el gráfico 2 se muestran estas mismas distancias ideológicas con la madre. Los datos muestran que también existe una distancia muy corta entre el posicionamiento político propio y el de la madre. Del mismo modo, la media de las distancias es inferior a la unidad en todos los años y un porcentaje alrededor del 40 por 100 comparte la ubicación ideológica con la madre.

13 En el año 1994 este porcentaje subía al 52 por 100, pero conviene matizar que su fiabilidad puede ser dudosa, debido al gran número de casos «missing» que se producen en esa encuesta concreta al calcular las distancias ideológicas. 


\section{GRÁFICO 1}

\section{Distancia ideológica con el padre}

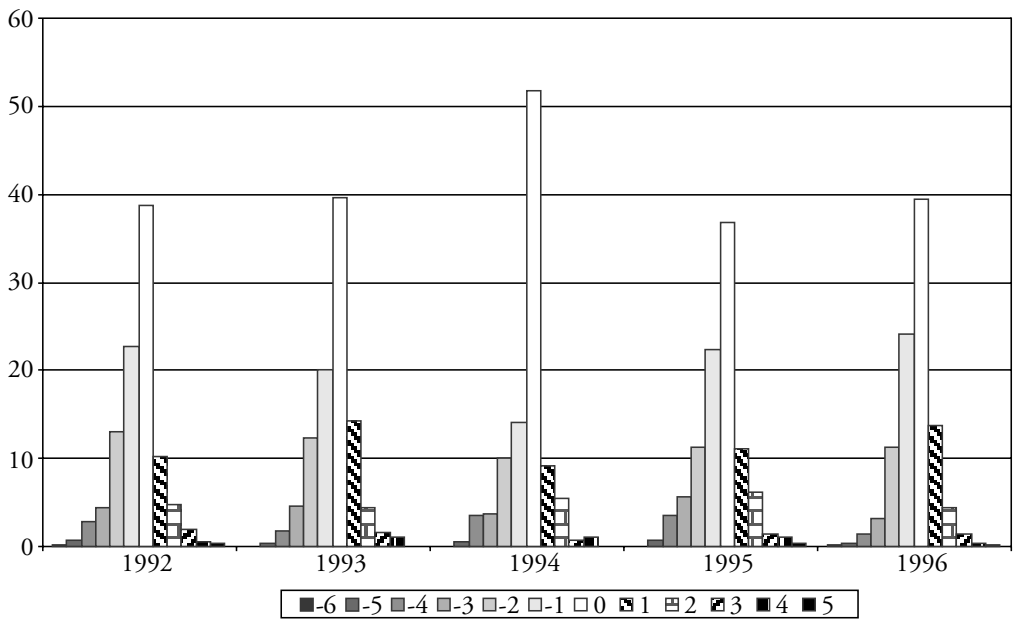

Fuente: Elaboración propia, a partir de las Encuestas de Cultura Política y Economía del CIRES (1992-1996).

\section{GRÁFICO 2}

Distancia ideológica con la madre

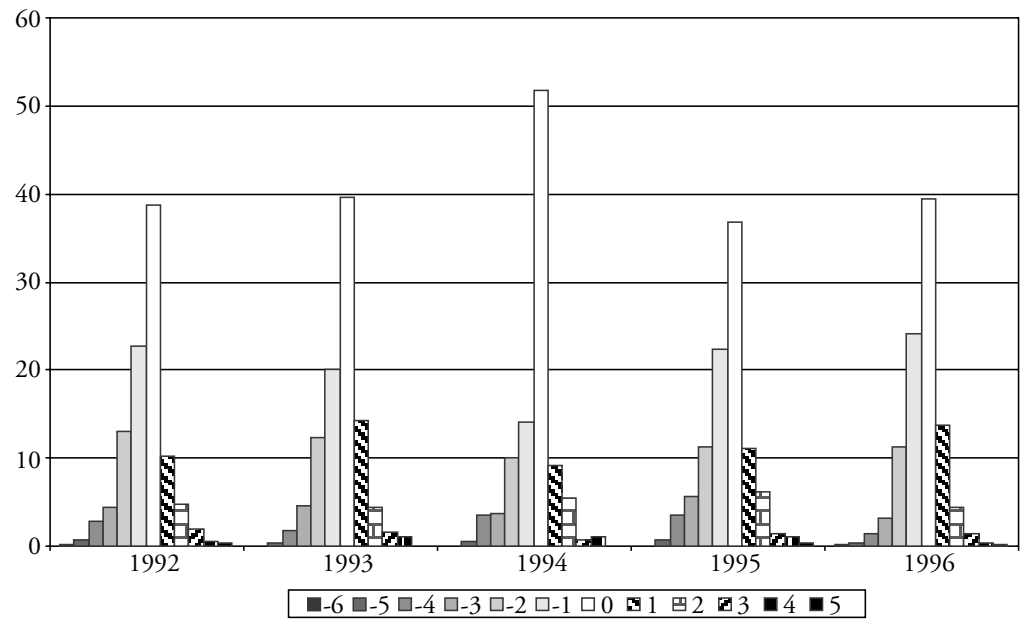

Fuente: Elaboración propia, a partir de las Encuestas de Cultura Política y Economía del CIRES (1992-1996). 
Aunque las distancias ideológicas con ambos progenitores son bastante reducidas, se observa una ligera tendencia de las nuevas generaciones a situarse ligeramente más a la izquierda que sus padres. Puesto que, según revelan diversos estudios sobre la juventud española, los jóvenes se sitúan más a la izquierda que sus padres, es probable que esta impresión perdure en la época adulta. Se trata más bien de una deformación de la percepción de la posición ideológica de la familia antes que de una distancia ideológica real. De lo contrario habría que esperar que cada generación se situase progresivamente más a la izquierda que la anterior. Y lo cierto es que sucede justo lo contrario. Durante los años noventa los jóvenes españoles han pasado a situarse ligeramente a la derecha de la posición que ocupaban los que eran jóvenes a principios de los años ochenta.

Otro dato que proporciona el cálculo de la distancia ideológica es el progenitor al que los sujetos se sienten más próximos ideológicamente. En principio, como puede verse en el gráfico 3, la inmensa mayoría de ellos se sienten igual de próximos a ambos progenitores (un 87 por 100 en 1996 y porcentajes similares para el resto de años). Por otra parte, el porcentaje de los que se sienten más próximos al padre es similar al de los que se sienten más próximos a la madre, del mismo modo que no existen diferencias sustanciales en las medias de la distancia ideológica con respecto a cualquiera de los progenitores.

En cualquier caso estas pautas generales sobre el conjunto de la población española dicen poco sobre las dinámicas de socialización, puesto que no tienen en cuenta los diferentes factores, que pueden influir en la transmisión de orientaciones ideológicas de unas generaciones a otras. Por este motivo se ha controlado la influencia de ciertas variables sobre la distancia ideológica con cada uno de los progenitores: el sexo, la generación, el nivel educativo, el nivel de práctica religiosa, la ideología y la clase social subjetiva. Por último se ha querido indagar la influencia de una variable típica en diversos estudios

\section{GRÁFICO 3}

\section{Progenitor al que se siente más lejano ideológicamente (1996)}

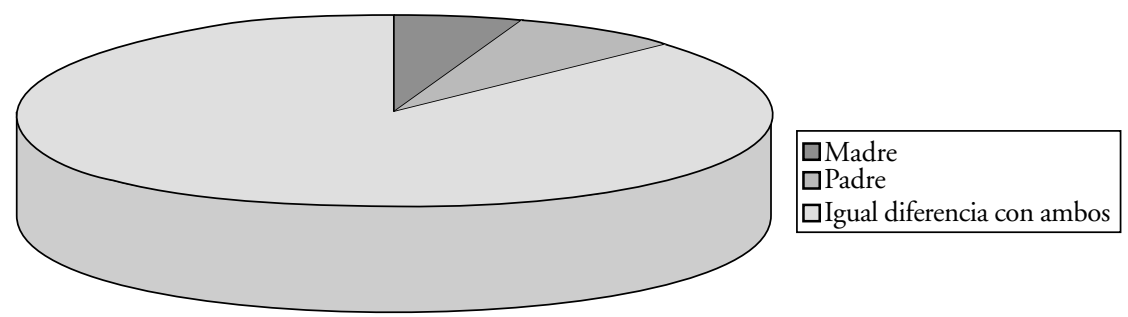

Fuente: Elaboración propia, a partir de las Encuestas de Cultura Política y Economía del CIRES (1992-1996). 
clásicos de socialización política, el nivel de discusión política en la familia de origen.

La interacción de la variable sexo con la distancia ideológica a cada uno de los progenitores resulta de sumo interés, ya que permite aproximarse a las dinámicas de socialización en el interior de la familia. Por una parte, los varones son los que se sitúan más lejos de ambos progenitores, mientras que las mujeres se sitúan siempre más cerca tanto del padre como de la madre. Por otro lado, tanto para hombres como para mujeres, la media de la distancia ideológica con el padre es ligeramente inferior a la media de la distancia ideológica con la madre, puesto que las nuevas generaciones tienden a situarse a la izquierda de las precedentes y los hombres suelen ubicarse a la izquierda de las mujeres. No obstante, ya se ha mencionado que la ideología de los españoles covaría más estrechamente con la ideología materna que con la paterna.

Esta relación más estrecha con la ideología de la madre se explicaría por la presencia más intensa de la madre durante el proceso de socialización primaria. El modelo de autoridad paterna en la familia tradicional no significa que la ideología del padre sea la dominante en los hijos. Como concluye Abramson (1983), después de examinar los principales modelos de socialización política, la ideología se transmite a través de mecanismos más sutiles que no implican adoctrinamiento ni imposición expresa de normas. Debido a que los niños adquieren las identificaciones ideológicas a edades muy tempranas, en las que tienen mayor contacto con la madre, cabe esperar que las madres estén en mejor disposición de controlar esos sutiles mecanismos por los que los niños aprenden una orientación ideológica determinada, a pesar de que no conozcamos bien cuáles son y cómo operan esos mecanismos.

TABLA 2

Grupos con media de distancia ideológica menor a ambos progenitores*

\begin{tabular}{|c|c|c|}
\hline & Padre & Madre \\
\hline 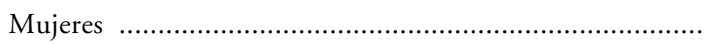 & 0,28 & 0,31 \\
\hline 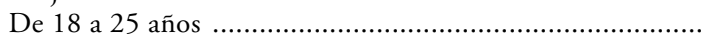 & 0,29 & 0,24 \\
\hline Estudios primarios & 0,26 & 0,35 \\
\hline 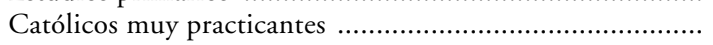 & 0,05 & 0,35 \\
\hline Ideología política de centro & 0,00 & 0,10 \\
\hline 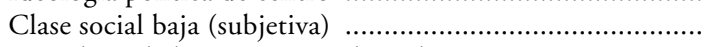 & 0,23 & 0,21 \\
\hline 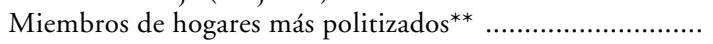 & 0,07 & 0,21 \\
\hline TOTAL ....................... & 0,34 & 0,39 \\
\hline
\end{tabular}

* Distancias en valor absoluto.

** Individuos en cuyo hogar materno se hablaba mucho de política a la edad de dieciséis años.

Fuente: Elaboración propia, a partir de la Encuesta de Cultura Política y Económica del CIRES (1996). 
En este contexto, el modelo de autoridad paterna puede provocar reacciones de rechazo o aversión en los individuos. Como subrayan Bahr, Déchaux y Stiehr (1995), la tendencia en las sociedades occidentales es, precisamente, hacia la reducción de la autoridad paterna por oposición al fortalecimiento de los vínculos que unen a las madres con los hijos. Sería de suma importancia, por tanto, poder estudiar a largo plazo cómo la progresiva incorporación de la mujer al mercado de trabajo, así como la mayor dedicación de los varones a las prácticas de crianza, pueden alterar este panorama.

Por lo que respecta a la edad, se observa que en el grupo de dieciocho a veinticinco años las distancias ideológicas, tanto con respecto al padre como a la madre, son menores que en los estratos de mayor edad. La mayor diferencia ideológica con los progenitores tiende a darse en los grupos de edad de veintiséis a cuarenta años y de cuarenta y uno a sesenta años, al tiempo que se reduce en el grupo de los que tienen más de sesenta años.

Este hecho está relacionado con las condiciones de socialización de las distintas generaciones y el conflicto generacional presente en cada uno de estos contextos. Quienes ahora están en la cuarentena, nacieron a fines de los años cincuenta y principios de los sesenta. Su infancia y primera juventud se desarrollaron en el contexto de los profundos cambios que, tanto desde el punto de vista estrictamente sociológico como económico y político, vivió la sociedad española en la década de los sesenta y setenta. Los procesos de cambio social son propicios para la emergencia de conflictos generacionales, por efecto del distinto ritmo de adaptación a los cambios, que es más rápido en los grupos más jóvenes y más lento en los grupos de más edad. Consecuentemente, la distancia ideológica entre las generaciones tiende a aumentar.

Por lo que se refiere al nivel educativo, se aprecia que la distancia ideológica tiende a aumentar conforme se incrementa el nivel de formación, un fenómeno que es paralelo tanto para la distancia con el padre como con la madre. Esta cuestión nos sitúa directamente ante el papel que juegan otras instituciones sociales (el sistema educativo, en este caso) en el proceso de socialización política. Parece ser que conforme crece el nivel educativo, los individuos tienden a adquirir un juicio político más independiente de su familia de origen. La educación contribuye al desarrollo de la capacidad crítica y, por tanto, a la independencia ideológica. Lo cual no implica necesariamente que el sistema educativo marque una dirección determinada en el posicionamiento ideológico de los individuos.

El nivel de práctica religiosa de los sujetos marca unas claras diferencias en cuanto a la distancia ideológica con ambos progenitores. Los grupos de mayor práctica religiosa son los que se sienten ideológicamente más cercanos a su familia de origen, al tiempo que la distancia crece en sentido inversamente proporcional a la práctica religiosa, siendo los más distantes a sus padres los que se consideran indiferentes en materia religiosa. La religiosidad contribuye, por tanto, a que la transmisión de las orientaciones ideológicas sea más efectiva en el seno de la familia, lo cual se corresponde con el énfasis de la religión cristiana en la obediencia paterna. 
Otra variable que influye de forma considerable en la distancia ideológica con respecto a ambos progenitores es la propia posición ideológica del sujeto. La distancia ideológica crece de forma bastante más rápida conforme los individuos se sitúan más a la izquierda. Los que se definen como de derechas y, particularmente, los que se sitúan en el centro ideológico son los menos distantes de sus padres en cuestiones ideológicas.

La influencia de la clase social subjetiva sobre las distancias ideológicas con ambos progenitores no tiene una tendencia definida. No obstante, los individuos que se dicen de clase baja tienden a mostrar las menores distancias ideológicas con respecto a ambos progenitores, mientras que el grupo con mayor distancia ideológica es el de la clase media y media baja, y el grupo de clase media-alta suele situarse en una posición intermedia.

La última variable analizada es la frecuencia de discusión política en el hogar familia cuando el sujeto tenía la edad de dieciséis años. Conforme a la evidencia derivada de los primeros estudios de socialización política, cabría esperar que ésta fuese una variable con una gran influencia sobre la distancia ideológica sentida con el padre y la madre. Cuanto mayor sea la comunicación política, mejores son las condiciones para la transmisión de lealtades ideológicas de una generación a otra.

No obstante, los datos analizados aquí para el caso español muestran una escasa capacidad discriminante de esta variable sobre la distancia ideológica con los progenitores. El punto de corte se produce entre quienes dicen que en su hogar familiar se hablaba mucho de política y el resto. Los primeros se encuentran bastante más cercanos a sus dos progenitores. Sin embargo, las diferencias entre quienes manifiestan que en su casa se hablaba muy poco, poco, regular o bastante de política no son especialmente significativas, ni estables a lo largo del tiempo.

\section{CONCLUSIONES}

El análisis de los datos empíricos sobre la similitud/diferencia ideológica entre padres e hijos muestra, en primer lugar, que se da un alto grado de coincidencia. Los hijos suelen compartir con sus progenitores, padre y madre, una de las actitudes políticas básicas en nuestra cultura, la ubicación en el continuum ideológico izquierda-derecha. Por otra parte, los indicadores sobre consenso normativo en cuestiones políticas revelan que los jóvenes comparten un buen número de normas políticas con sus padres, a pesar de que en la familia española se habla poco de política, un fenómeno que no es exclusivo de la sociedad española, sino que se da también en otros países del entorno.

Las correlaciones halladas entre la posición ideológica de los españoles y la que éstos atribuyen a sus padres también se encuentran en valores similares a los de otros países mediterráneos como Francia e Italia. En este proceso de acercamiento ideológico entre las generaciones influyen distintos factores. 
Tanto padres como hijos han experimentado un desplazamiento hacia el centro del espectro ideológico desde sus respectivas posiciones (más a la derecha los padres, más a la izquierda los hijos), producto de las grandes transformaciones que ha vivido la sociedad española en las últimas décadas.

A pesar de que esas transformaciones también han afectado de forma radical a la familia, ésta continúa siendo una instancia eficaz en el proceso de socialización política. El papel asumido en el proceso de socialización por otras instancias especializadas, como la escuela o los medios de comunicación, no han erosionado la influencia de la familia de origen sobre las orientaciones ideológicas de los españoles. El grueso de los entrevistados entre 1992 y 1996 en las encuestas de cultura política y económica del CIRES se sitúan a una distancia ideológica bastante reducida tanto del padre como de la madre, si bien existe una suave tendencia a situarse ligeramente más a la izquierda que los progenitores.

Los principales resultados muestran una amplia congruencia con los estudios ya clásicos de socialización política llevados a cabo en otros países. Padres e hijos comparten las normas políticas fundamentales, que son transmitidas durante la infancia temprana en contacto con la familia. La cantidad de normas transmitidas y la calidad de la transmisión dependerá de ciertos elementos ambientales en los que tiene lugar el proceso de socialización y que están relacionados con los procesos de cambio social que vive una sociedad en una época histórica determinada.

En último lugar, existen distintas variables que influyen en la distancia ideológica percibida con respecto a ambos progenitores, las cuales actúan en sentido paralelo tanto con respecto a la distancia ideológica con respecto al padre como a la madre. Sin embargo, por encima de las diferencias se impone un patrón bastante similar en cuanto a la transmisión de identidades ideológicas en el seno de la familia española, lo cual se corresponde con el hecho de ser una institución central en distintos ambientes sociales.

A pesar del hecho constatado de que la familia juega un papel decisivo en la transmisión de las lealtades ideológicas, existe aún muy poco conocimiento sobre cómo se desarrolla este proceso. El proceso de socialización política en la familia continúa siendo una "caja negra». Mucha más información sería necesaria acerca de cómo las transformaciones que ha experimentado la familia española en la segunda mitad del siglo XX han influido en las pautas de socialización. Igualmente, se requeriría investigación sobre el papel que los medios de comunicación, y crecientemente las nuevas tecnologías de la información, están ejerciendo en la socialización política durante la infancia. Para abordar estas cuestiones sería preciso un gran volumen de investigación cualitativa al respecto. 


\section{REFERENCIAS BIBLIOGRÁFICAS}

Abramson, P. R. (1983): Political attitudes in America. Formation and change, W. H. Freeman and Company, San Francisco.

ANDrÉs Orizo, F. (1996): Sistemas de valores en la España de los 90, CIS, Madrid.

ARistóteles (1991): Política, Alianza, Madrid.

Bahr, M.; DéchauX, J. K., y STIEhr, K. (1995): «Evolución de los vínculos familiares: Padres e hijos adultos», en S. Langlois y S. del Campo (eds.), ¿Convergencia o divergencia? Comparación de tendencias sociales recientes en las sociedades industriales, Fundación BBV, Bilbao, pp. 153-219.

Barnes, S. H.; KaAse, M., et al. (1979): Political action: Mass participation in five Western Democracies, Sage Publications, Beverly Hills.

BELL, D. (1994): Las contradicciones culturales del capitalismo, Alianza, Madrid.

Campbell, A.; Converse, P. E.; Miller, W. E., y Stokes, D. E. (1960): The American voter, Wiley, New York.

CAPLOW, T. (1995): «La reducción de la autoridad personal», en S. Langlois y S. del Campo (eds.), ¿Convergencia o divergencia? Comparación de tendencias sociales recientes en las sociedades industriales, Fundación BBV, Bilbao, pp. 273-285.

CASTILlo, P. DEL (1990): "Aproximación al estudio de la identificación partidista en España», en Revista de Estudios Políticos, núm. 70, pp. 125-141.

CIS (1999): Datos de Opinión, CIS, núm. 19.

Connel, R. W. (1972): «Political socialization in the American family: The evidence re-examined», en Public Opinion Quarterly, núm. 25, pp. 323-333.

Converse, P. E., y Dupeux, G. (1962): «Politicization of the electorate in France and the United States», en Public Opinion Quarterly, núm. 26, pp. 1-23.

DAVIES, J. C. (1970): «The family's role in political socialization», en R. S. Sigel, Learning about politics. A reader in political socialization, Random House, New York.

Dawson, R. E.; PrewITt, K., y DaWson, L. S. (1977): Political socialization, Little, Brown and Company, Boston.

Dowse, R. E., y Hughes, J. A. (1971): "The family, the school, and the political socialization Process», en Sociology, núm. 5, pp. 21-45.

- (1999): Sociología política, Alianza, Madrid.

EASTON, D., y DENNIS, J. (1967): "The child's acquisition of regime morms: Political efficacy», en American Political Science Review, núm. 61, pp. 25-38.

- (1969): Children in the political system: Origins of political legitimacy, McGraw-Hill, New York.

Elzo, J., et al. (1994): Jóvenes españoles 94, Fundación Santa María, Madrid.

- (1999): Jóvenes españoles 99, Fundación Santa María, Madrid.

Greenstein, F. I. (1965): Children and politics, Yale University Press, New Haven.

Greenstein, F. I., y Tarrow, S. (1970): Political orientations of children: The use of a semi-projective technique in three nations, Sage Publications, Beverly Hills.

Halman, L., y MOOR, R. DE (1994): «Individualización y cambio de valores en Europa y Norteamérica», en J. Díez Nicolás y R. Inglehart (eds.), Tendencias mundiales de cambio en los valores sociales y politicos, Fundesco, Madrid, pp. 29-62.

Harrop, M., y Miller, W. L. (1990): Elections and voters. A comparative introduction, Macmillan Education, Londres.

Hess, R. D., y Torney, J. V. (1965): The development of basic attitudes and values toward Government and Citizenship during the elementary school years, University of Chicago Press, Chicago.

Hess, R. D., y Torney, J. V. (1967): The development of political attitudes in children, Aldine, Chicago.

Hyman, H. H. (1959): Political socialization: A study in the psychology of political behavior, Free Press, Glencoe. 
IGLesias de Ussel, J. (1990): «La familia y el cambio político en España», en Revista de Estudios Políticos, núm. 67, pp. 235-259.

- (1998): Familia y cambio político en España, Tecnos, Madrid.

Instituto DE LA JUVENTUd (1991): Actitudes políticas de la juventud en España, Instituto de la Juventud, Madrid.

Jaime Castillo, A. M. (1998): «El perfil del votante español: Análisis de las elecciones de 1996», Comunicación presentada en el VI Congreso de Sociología de la FES, A Coruña (inédita).

Jennings, M. K., y LANGTON, K. P. (1969): «Mothers and fathers: The formation of political orientations among young Americans», en Journal of Politics, núm. 31, pp. 329-358.

Jennings, M. K., y Niemi, R. G. (1968): «The transmission of political values from parent to child», en American Political Science Review, núm. 62, pp. 169-184.

- (1974): The political character of adolescence: The influence of families and schools, Princeton University Press, Princeton.

- (1981): Generations and politics: A panel study of young adults and their parents, Princeton University Press, Princeton.

Jennings, M. K.; Allerbeck, K. R., y Ronsenmayr, L. (1979): "Generations and families: General orientations», en S. H. Barnes, M. Kaase et al., Political action: Mass participation in five Western Democracies, Sage Publications, Beverly Hills, pp. 449-486.

LANE, R. E. (1959): "Fathers and sons. The foundations of political beliefs», en American Sociological Review, núm. 24, pp. 502-511.

Maravall, J. M. (1985): La politica de la transición, Taurus, Madrid.

Merton, R. K. (1986); «Estructura social y anomia. Revisión y ampliación», en E. From, M. Horkheimer, T. Parsons et al., La familia. Editorial Península, Madrid, pp. 67-122.

Montero, J. R. (1996): «Elecciones y comportamiento electoral: Dimensiones, factores y reglas», en J. Tusell, E. Lamo de Espinosa y R. Pardo (eds.), Entre dos siglos. Reflexiones sobre la Democracia Española, Alianza, Madrid, pp. 181-226.

Murillo Ferrol, F. (1987): «La familia y el proceso de socialización», en F. Murillo Ferrol, Ensayos sobre sociedad y política, Editorial Península, Madrid, pp. 297-315.

Nie, N. H.; Verba, S., y PetrociK, J. R. (1976): The changing American voter, Harvard University Press, Cambridge.

OrtegA, F. (1975): «La familia, unidad socializadora», en Instituto de Sociología Aplicada de Madrid, Estudio sociológico de la familia española, CECA, Madrid, 171-208.

PARSONS, T. (1986): «La estructura social de la familia», en E. From, M. Horkheimer, T. Parsons et al., La Familia, Editorial Península, Madrid, pp. 31-65.

Parsons, T., y Bales, R. F. (1955): Family, socialization and interaction process, The Free Press, Glencoe.

Percheron, A. (1990): "Production et transmission des valeurs au sein de la famille», en VV. AA., Du politique et du social dans l'avenir de la famille, La Documentation Française, París, pp. 131-139.

- (1991): «Les valeurs familiales», en A. Singly (dir.), Famille, l'état des savoirs, Editions de la Découverte, París, pp. 183-193.

Percheron, A., y Jennings, M. K. (1981): «Political continuities in French families», en Comparative Politics, núm. 13, pp. 421-436.

Rodríguez, A. (1988): «Socialización política», en J. Seoane y A. Rodríguez (eds.), Psicología política, Pirámide, Madrid.

Sabucedo, J. M. (1996): Psicología Política, Síntesis, Madrid.

SChWARTZ, S. K. (1975); "Preschoolers and politics», en D. C. Schwartz y S. K. Schwartz, New directions in political socialization, Free Press, Glencoe.

Tedin, K. L. (1974): "The influence of parents on the political attitudes of adolescents», en American Political Science Review, núm. 68, pp. 1579-1592.

Tezanos, J. F. (1982): Sociología del socialismo español, Tecnos, Madrid. 


\begin{abstract}
Basic political socialization takes place within the family. Classical political socialization studies that have been carried out have shown that children tend to reproduce the ideological leanings of their parents. Available data for Spain shows a great similarity between the political position of the present generation and its predecessor. In this respect, it should be pointed out that despite the transformations that the Spanish family has undergone over the last few decades, the family continues to be an efficient agent in the political socialization process. Lastly, an analysis is made of the influence that some variables have over the ideological distancing of parents and children.
\end{abstract}

